\title{
Conocimientos, actitudes y prácticas sobre el Zika en estudiantes de medicina, 2016.
}

\author{
Knowledge, attitudes and practices on medical students in Zika, 2016. \\ Rios-González Carlos Miguel ', De Benedictis-Serrano Ginno Alessandro 2, Chirino-Caicedo \\ Alfonzo David ${ }^{2}$
}

\section{RESUMEN}

Objetivo: Determinar los conocimientos, actitudes y prácticas sobre el Zika en estudiantes de medicina de la Universidad de Carabobo, sede Aragua en Venezuela, durante los meses de marzo a abril de 2016.

Material y métodos: Se realizó un estudio observacional, descriptivo, de corte transversal, con muestreo no probabilístico por conveniencia en estudiantes de medicina de la Universidad de Carabobo, sede Aragua en Venezuela, durante los meses de marzo a abril de 2016. Se diseñó y validó un cuestionario para la recolección de los datos. Los datos se expresan en tabla de frecuencia y proporciones.

Resultados: De 172 estudiantes de medicina, de 18 a 41 años de edad, con una media de 20,64 años (DE=2,26), de los cuales el $66,28 \%$ (II4) perteneció al sexo masculino, el nivel de conocimiento fue en $90,70 \%$ (I56) alto. El $56,98 \%$ (98) de los estudiantes están de acuerdo en que el público tiene el papel más importante en el control del Zika. En cuanto a las prácticas; el 75,58\% (130) de los estudiantes de medicina no usan repelentes en ningún momento del día; $20,35 \%$ (35) solo lo usa por las noches.

Conclusión: El nivel de conocimiento general de los estudiantes fue alto, una actitud en la mayoría de los casos buena y prácticas no tan buena.

\section{ABSTRACT}

Objective: Determine the knowledge, attitudes and practices about Zika medical students at the University of Carabobo in Venezuela, Aragua headquarters, during the months of March and April 2016.

Material and Methods: A descriptive cross-sectional study with non-probabilistic sampling was performed for convenience in medical students from the University of Carabobo in Venezuela, Aragua headquarters during the months of March and April 2016. It was designed and validated a questionnaire to data collection. Data is expressed in frequency table and proportions.

Results: Of 172 medical students, 18 to 4 I years old, with an average of 20,64 years (SD=2,26), of which $66,28 \%$ (I I4) belonged to the male, the level of knowledge was $90,70 \%$ (I56) high. The $56,98 \%$ (98) of the students agree that the public has the most important role in controlling Zika. As for the practices; 75,58\% (130) of medical students do not use repellents at any time of the day; 20,35\% (35) uses it only at night.

Conclusion:The level of general knowledge of students was high, a good attitude in most cases and not so good practices.

\section{INTRODUCCIÓN}

$\mathrm{E}^{1}$ Zika es considerado una enfermedad emergente en distintos países del continente americano. Desde su primera notificación en el año 2015, se estima que se han producido cerca de un millón de casos, y 22 países de América y el Caribe informan casos de transmisión autóctona. Al igual que el Dengue y el Chikungunya es transmitido por un vector, el Aedes; debido a la alta distribución de este vector, casi todos los países de América presentan estas infecciones ${ }^{1,2}$.

El hábitat del Aedes aegypti puede aparecer cuando cesan las lluvias y se presentan días soleados o llega el verano; las zonas inundadas, especialmente en regiones tropicales, al disminuir los niveles del agua dejarán grandes y extensas zonas ya con agua clara o fangosa, hábitat ideal para el crecimiento de los vectores ${ }^{3}$.
VB

El virus ha demostrado ser altamente neurotrópico en modelos animales, infectando el cerebro y produciendo degeneración neuronal; sin embargo, la observación más preocupante es el aumento significativo de casos de microcefalia y malformaciones del sistema nervioso central en recién nacidos, informados en el noreste de Brasil desde octubre del 2015 $5^{4,5}$. Actualmente no existe tratamiento antiviral o vacuna específica para a infección por el virus del Zika (ZIKV). Lo recomendable es el tratamiento sintomático posterior a la exclusión de enfermedades más graves; la lucha vectorial es la única forma de evitar la propagación de esta enfermedad 6 .

Por todo lo anterior se ha planteado como objetivo
${ }^{1}$ Facultad de Ciencias Médicas, Universidad Nacional de Caaguazú, Cnel. Oviedo, Paraguay.

2 Facultad de Ciencias de la Salud, Universidad de Carabobo Sede Aragua. Venezuela.

Correspondencia a: Carlos Miguel Rios González carlosmigue_rios@live.com

Palabras clave: Zika Virus; Actitud; Conocimiento; Estudiantes de medicina..

Keywords: Virus; Attitude; Knowledge; Medicine students.

Procedencia y arbitraje: no comisionado, sometido a arbitraje externo.

Recibido para publicación: 27 de Agosto del 2016 Aceptado para publicación: 06 de Noviembre del 2016

Citar como:

Rev Cient Cienc Med 2016;19(2): 33 - 37 
determinar los conocimientos, actitudes y prácticas sobre el Zika en estudiantes de medicina de la Universidad de Carabobo, sede Aragua en Venezuela, durante los meses de marzo a abril de 2016.

\section{MATERIALES Y MÉTODOS}

Diseño de estudio y área: Se realizó un estudio observacional, descriptivo, de corte transversal.

Población y muestra: El universo lo constituyeron 412 estudiantes; sin embargo, fueron incluidos 172 estudiantes de la carrera de medicina de primer a quinto año de la Universidad de Carabobo, sede Aragua en Venezuela, durante los meses de marzo a abril de 2016; con un muestreo no probabilístico por conveniencia. A todos los participantes del estudio se les notificó de los objetivos del estudio, y aquellos que estuvieron de acuerdo procedieron al llenado del cuestionario electrónico mediante Google Doc $@$.

Criterios de inclusión y exclusión: Fueron incluidos los estudiantes de medicina de la Universidad de Carabobo, sede Aragua en Venezuela, durante los meses de marzo a abril de 2016 que hayan accedido a llenar el cuestionario. Fueron excluidos todos aquellos estudiantes que no desearon participar del estudio y aquellos que no hayan llenado las variables sociodemográficas.

Instrumento: Se diseñó un cuestionario, que contenía 23 preguntas, divididas en 4 secciones: epidemiologia, conocimientos, actitudes y prácticas. Se incluyeron 8 preguntas de conocimiento que miden los niveles del estudiante con respecto a la enfermedad del Zika, 5 preguntas que miden actitud y 5 preguntas que miden las prácticas de prevención de la enfermedad.

Validación y confiabilidad del instrumento:

-Cualitativo: Se realizó con un epidemiólogo y un infectólogo, quienes evaluaron todas las preguntas. Se utilizó el método Delphi.

-Cuantitativo: Se realizó un estudio piloto en 40 estudiantes de medicina de primer a quinto año de la carrera de medicina de la Universidad de Carabobo, sede Aragua en Venezuela, los cuales fueron excluidos de la muestra final.

Aspectos éticos: Toda la información recogida por los investigadores fue tratada con confidencialidad, igualdad y justicia; no se divulgó ni repitió ninguno de los resultados.

Análisis estadístico: Todos los datos proveídos por los cuestionarios fueron cargados a Microsoft Office Excel 2013@), y luego del control de calidad fueron exportados para su análisis en Stata 14.0. Los datos se expresan en tablas de frecuencias, medidas de tendencia central y dispersión. Para el análisis de confiabilidad se obtuvo con el coeficiente de Alfa de Cronbach (valor mayor de 0,7 define una confiabilidad aceptable).

\section{RESULTADOS}

Fueron incluidos en el estudio 172 estudiantes de medicina, de 18 a 41 años de edad, con una media de 20,64 años ( $\mathrm{DE}=2,26)$, de los cuales el 66,28\% (114) perteneció al sexo masculino, siendo de los cursos tercero en $49,42 \%$ (85) y segundo en $17,44 \%$ (30) (Ver Tabla 1).

El nivel de conocimiento fue en $90,70 \%$ (156) alto y 9,30\% (16) un nivel medio (Ver Tabla 2)

Con respecto a las actitudes; el 56,98\% (98) de los estudiantes están de acuerdo en que el público tiene el papel más importante en el control del Zika, el 54,07\% (93) está totalmente de acuerdo en que está en riesgo de contraer infección por Zika, mientras que el 1,74\% (3) está totalmente en desacuerdo. El 57,56\% (99) de los estudiantes están totalmente de acuerdo en que la infección por el Zika se puede prevenir, 37,79\% (65) está indeciso y/o dudoso respecto a si el Zika es una infección mortal (Ver Tabla 3).

En cuanto a las prácticas; 75,58\% (130) de los estudiantes de medicina no usan repelentes en ningún momento del día y 20,35\% (35) solo lo usa por las noches. En el caso de fiebre: $80,91 \%$ (139) da paracetamol en caso de síndrome febril en el hogar y el 16,28\% (28) acude a un médico de inmediato. 50\% (86) de los estudiantes de medicina afirman limpiar su casa y alrededores más de 2 veces por semana.

El 73,26\% (126) de los estudiantes nunca han participado de una actividad ambiental (Ver Tabla 4).

DISCUSIÓN

Como es conocido, el ZIKV es uno de los arbovirus con mayor circulación durante el 2015 y el 2016 en toda América y es considerada un grave problema para la salud publica debido a sus efectos en las embarazadas ${ }^{6,7}$, por lo que los conocimientos, las actitudes y las prácticas por parte de los estudiantes de medicina es indispensable para la lucha contra esta infección. Si bien la infección por este virus aparenta ser reciente 8 y probablemente en muchas mallas curriculares no haya sido incluida aún su estudio, este paradigma reciente no ha sido evidenciado en este estudio, puesto que el mayor porcentaje de los estudiantes presentaron un nivel de conocimiento alto, un pequeño porcentaje un nivel de conocimiento medio y ninguno con nivel de conocimiento bajo; esto podría ser que -a pesar de no ser incluido dentro de las mallas curriculares- el acceso a la información es mucho fácil debido a los medios de comunicación, el internet, y también es importante destacar el papel que cumple las redes sociales ${ }^{9}$, donde pulula la información respecto a esta infección. La pregunta con mayor falla respecto al conocimiento fue sobre la sintomatología, donde los estudiantes referían la 


\begin{tabular}{|c|c|c|}
\hline & $\mathbf{N}$ & $\%$ \\
\hline \multicolumn{3}{|l|}{ Sexo } \\
\hline Masculino & 114 & 66,28 \\
\hline Femenino & 58 & 33,72 \\
\hline \multicolumn{3}{|c|}{ Año cursado actualmente } \\
\hline Primer curso & 28 & 16,28 \\
\hline Segundo curso & 30 & 17,44 \\
\hline Tercer curso & 85 & 49,42 \\
\hline Cuarto curso & 14 & 8,14 \\
\hline Quinto curso & 15 & 8,72 \\
\hline
\end{tabular}

Tabla 2: Distribución según el nivel de conocimiento de los estudiantes de medicina de la Universidad de Carabobo, sede Aragua. Venezuela, 2016.

$\begin{array}{lll} & \text { N } & \% \\ \text { Nivel de conocimiento } & & \\ \text { Alto } & 156 & 90,70 \\ \text { Medio } & 16 & 9,30 \\ \text { Bajo } & 0 & 0\end{array}$

Tabla 3: Distribución según las actitudes de los estudiantes de medicina de la Universidad de Carabobo, sede Aragua. Venezuela, 2016.

\begin{tabular}{llllll} 
& $\begin{array}{l}\text { Totalmente } \\
\text { en } \\
\text { desacuerdo }\end{array}$ & $\begin{array}{l}\text { En } \\
\text { desacuerdo }\end{array}$ & $\begin{array}{l}\text { Indeciso/ } \\
\text { Dudoso }\end{array}$ & De acuerdo & $\begin{array}{l}\text { Totalmente } \\
\text { de acuerdo }\end{array}$ \\
\hline $\begin{array}{l}\mathbf{N}(\%) \\
\text { ¿El público tiene el papel más }\end{array}$ & 0 & $\mathbf{N}(\%)$ & $\mathbf{N}(\%)$ & $\mathbf{N}(\%)$ & $\mathbf{N}(\%)$ \\
$\begin{array}{l}\text { importante en el control del } \\
\text { Zika? }\end{array}$ & $2(1,16)$ & $23(13,37)$ & $98(56,98)$ & $49(28,49)$ \\
$\begin{array}{l}\text { ¿Cree usted que está en riesgo } \\
\text { de contraer Zika? }\end{array}$ & $3(1,74)$ & $2(1,16)$ & $6(3,49)$ & $68(39,53)$ & $93(54,07)$ \\
$\begin{array}{l}\text { ¿Cree usted que la infección } \\
\text { por Zika se puede prevenir? }\end{array}$ & 0 & $1(0,58)$ & $7(4,07)$ & $65(37,79)$ & $99(57,56)$ \\
¿Es la infección por Zika una \\
enfermedad mortal?
\end{tabular}

son los responsables de la propagación del Zika?

disnea, la tos y la hemorragia como sintomatologías frecuentes ${ }^{10}$.

Las actitudes en su mayoría fueron favorables: concuerdan que el público en general tiene el papel más importante en la prevención de esta infección, puesto que la lucha vectorial es una de las mejores estrategias para evitar la propagación de esta enfermedad11; es importante destacar a su vez el concepto de que los estudiantes están totalmente de acuerdo en que están en riesgo de adquirir la infección como cualquier otra persona; sin embargo, existen un pequeña minoría que no se considera susceptible para adquirir esta infección. Otro punto interesante es que, en su gran ma- yoría, los estudiantes se encontraban indecisos y dudosos sobre si la infección por este virus era mortal; y como es reportada por varios autores, esta infección no es mortal ${ }^{12,13}$.

En cuanto a las prácticas más referidas para evitar la infección son: la limpieza de casa y alrededores más de 2 veces por semana; sin embargo, un punto que llamo la atención es que la mayoría de los estudiantes de medicina no usaban repelentes y que no participan de trabajos colectivos para la limpieza de la comunidad, a no ser que sea exigido por las universidades, lo cual viene a mesa de debate sobre la importancia de las universidades en la lucha vectorial, puesto que estas 
prácticas están muy relacionadas con la continuidad en la cadena de transmisión no solo del Zika, sino también de otras arbovirosis como lo son el Dengue y el Chikungunya, y también las presentaciones endémicas de la enfermedad. Otra práctica que llamá la atención es que en su gran mayoría, ante algún caso probable de síndrome febril, los estudiantes administran el paracetamol o acetaminofén, que es una buena práctica según los protocolos 11; sin embargo, el control médico es indispensable para un buen diagnóstico y tratamiento oportuno.

En este estudio asimismo se pudo apreciar el gran interés por aprender sobre esta enfermedad una vez que conocen su gravedad y que las medidas de prevención están al alcance de sus manos.

La limitaciones del estudio se centran en que proviene de un muestreo no probabilístico, por lo que sería interesante encaminar otro estudio con aumen- to en el tamaño muestral, ajustes en el diseño y en lo posible a aplicado a todos los estudiantes de medicina de Latinoamérica a los efectos de medir el nivel de conocimiento real sobre esta infección, en los diferentes estratos.

A modo de conclusión se puede decir que el nivel de conocimiento general sobre la infección por Zika fue alto, las actitudes en su mayor parte fueron favorables; en cuanto a las prácticas no fueron muy buenas, puesto que a pesar del alto conocimiento que tiene los estudiantes sobre esta enfermedad, las medidas preventivas como el uso de repelente siguen siendo bajas, por lo que la capacitación continua en este tipo de enfermedades emergentes y reemergentes es imperativo para la formación de estudiantes capacitados para una buena salud pública.

\begin{tabular}{|c|c|c|}
\hline & $\mathbf{N}$ & $\%$ \\
\hline \multicolumn{3}{|l|}{ Uso de repelentes } \\
\hline Nunca & 130 & 75,58 \\
\hline Solo por la mañana & 4 & 2,33 \\
\hline Solo por la noche & 35 & 20,35 \\
\hline Todo el día & 3 & 1,74 \\
\hline \multicolumn{3}{|l|}{$\begin{array}{l}\text { En caso de alguien con fiebre en } \\
\text { casa, qué realiza }\end{array}$} \\
\hline Acude al médico. & 28 & 16,28 \\
\hline Da jugo de hoja de papaya. & 1 & 0,58 \\
\hline Da paracetamol & 139 & 80,91 \\
\hline Nada & 4 & 2,33 \\
\hline \multicolumn{3}{|l|}{$\begin{array}{l}\text { Con qué frecuencia limpia su casa y } \\
\text { alrededores }\end{array}$} \\
\hline Nunca & 4 & 2,33 \\
\hline Menos de 2 veces por semana & 43 & 25 \\
\hline Más de 2 veces por semana & 86 & 50 \\
\hline Todos los días & 39 & 22,67 \\
\hline \multicolumn{3}{|l|}{$\begin{array}{l}\text { Con qué frecuencia participa de } \\
\text { mingas ambientales }\end{array}$} \\
\hline Nunca & 126 & 73,26 \\
\hline Cada vez que la universidad lo exige & 33 & 19,19 \\
\hline Una vez al mes & 7 & 4,07 \\
\hline Todas las semanas & 6 & 3,49 \\
\hline \multicolumn{3}{|l|}{$\begin{array}{l}\text { Con respecto a las cubiertas y } \\
\text { botellas usted }\end{array}$} \\
\hline No hace nada & 84 & 48,84 \\
\hline Las quema & 3 & 1,74 \\
\hline $\begin{array}{l}\text { Las mete bajo un techo y pone boca } \\
\text { abajo }\end{array}$ & 27 & 15,70 \\
\hline Las entierra & 58 & 33,72 \\
\hline
\end{tabular}




\section{REFERENCIAS}

1- Vial PA, Araos RI. Virus Zika en un nuevo mundo. Rev Chil de Pediatr 2016; 87(2): 7981. Acceso: 10 de junio de 2016. Disponible en: http://www.sciencedirect.com/science/article/pii/ S0370410616000310

2- Rios C, Escobar J. Reflexiones sobre la infección por Zika en Paraguay. Rev Chil Infectol 2016; 33(2): 240-1. Acceso: 05 de junio de 2016. Disponible en: http://www.revista.sochinf.cl/ PDF_inf_2_2016/art19.pdf

3-Carvajal C, Peña O, Oletta F. Infección por virus Zika (VZIK): Arbovirosis emergente en las Américas. Med Interna (Caracas) 2015; 31(1): 8-15. Acceso: 10 de junio de 2016. Disponible en: http://medicinapreventiva.info/ medicina-interna/23034/infeccion-por-virus-zikavzik-arbovirosis-emergente-en-las-americas-porcarvajala09/

4- Rivera O. Aedes aegypti, virus dengue, chikungunya, zika y el cambio climático. Máxima alerta médica y oficial. Rev elect de Veter 2014; 15(10): 1-9. Acceso: 10 de junio de 2016. Disponible en: http://www.veterinaria.org/ revistas/redvet/n101014/101403.pdf

5- Ríos CM, Escobar JS. El embarazo y la infección por el virus del Zika: un problema emergente de la salud pública paraguaya. Pediatr (Asunción) 2016; 43(1): 77-8. Acceso: 05 de junio de 2016. Disponible en: http://scielo.iics. una.py/pdf/ped/v43n1/v43n1a10.pdf

6- Torres MA, Puerto F. Virus Zika, una nueva epidemia en puerta. Rev Bio med 2016; 27: 1-2. Acceso: 09 de junio de 2016. Disponible en: http://www.medigraphic.com/pdfs/revbio/bio2016/bio161a.pdf

7- Musso D, Aubry M, Broult J, Stassinopoulos A, Green J. Zika virus: new emergencies, potential for severe complications, and prevention of transfusion-transmitted Zika fever in the context of co-circulation of arboviruses. Blood Transfus 2016. Apr 28: 1-2. Disponible en: http://www.bloodtransfusion.it/ articolosing. aspx? id $=000816$

8- Ong CW. Zika virus: an emerging infectious threat. Intern Med J. 2016; 46(5): 525-30. Disponible en: http://onlinelibrary.wiley.com/ doi/10.1111/imj.13059/full

9- Fernández SM, Centellas FC. Investigar desde internet: Las redes sociales como abertura al cambio. Historia y comunicación social 2013; 18(esp): 663-75. Disponible en: http://revistas.ucm.es/index.php/HICS/article/ viewFile/44276/41834

10- Singh RK, Dhama K, Malik YS, Ramakrishnan MA, Karthik K, Tiwari R , et al. Zika Virus Emergence, evolution, pathology, diagnosis and control: current global scenario and future perspectives - A comprehensive review. Vet $Q$ 2016; 9: 1-43. Acceso: 09 de junio de 2016. Disponible en: https://www.researchgate. net/publication/296700595_Zika_Virus_Emergence_Evolution_Pathology_Diagnosis_ and_Control_Current_Global_Scenario_and_ Future_Perspectives_-_A_Comprehensive_ Review

11- Valerio L, Roure $\mathrm{S}$, Fernández G. Infección por el virus Zika o el futuro de las enfermedades infecciosas. Med Clin (Barc) 2016; 147(7): 300-5. Disponible en: http://fulltext. study/download/3794400.pdf

12- Wang Z, Wang $P$, An J. Zika virus and Zika fever. Virol Sin 2016; 31(2): 103-9. Disponible en: https://www.ncbi.nlm.nih.gov/ pubmed/27129450?dopt=Abstract

13- Nirav R. Soni. A new looming of Zika virus. APJR 2016; 5(3): 179-81. Disponible en: http://www.sciencedirect.com/science/article/pii/ S2305050016300392 